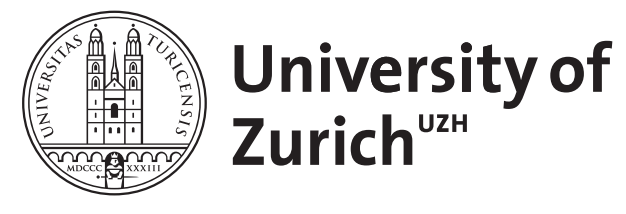

\title{
Flavour Physics and Implication for New Phenomena
}

\author{
Isidori, Gino
}

\begin{abstract}
Flavour physics represents one of the most interesting and, at the same time, less understood sector of the Standard Theory. On the one hand, the peculiar pattern of quark and lepton masses, and their mixing angles, may be the clue to some new dynamics occurring at high-energy scales. On the other hand, the strong suppression of flavour-changing neutral-current processes, predicted by the Standard Theory and confirmed by experiments, represents a serious challenge to extend the Theory. This article reviews both these aspects of flavour physics from a theoretical perspective.
\end{abstract}

DOI: https://doi.org/10.1142/9789814733519_0017

Posted at the Zurich Open Repository and Archive, University of Zurich ZORA URL: https://doi.org/10.5167/uzh-129889

Book Section

Originally published at:

Isidori, Gino (2016). Flavour Physics and Implication for New Phenomena. In: Malani, L; Rolandi, G. The Standard Theory of Particle Physics. Singapore: World Scientific Publishing, 339-355.

DOI: https://doi.org/10.1142/9789814733519_0017 


\title{
Chapter 1
}

\section{Flavour Physics and Implication for New Phenomena}

\author{
Gino Isidori \\ Physik-Institut, Universität Zürich, CH-8057 Zürich, Switzerland, \\ INFN, Laboratori Nazionali di Frascati, I-00044 Frascati, Italy
}

\begin{abstract}
Flavour physics represents one of the most interesting and, at the same time, less understood sector of the Standard Theory. On the one hand, the peculiar pattern of quark and lepton masses, and their mixing angles, may be the clue to some new dynamics occurring at high-energy scales. On the other hand, the strong suppression of flavour-changing neutral-current processes, predicted by the Standard Theory and confirmed by experiments, represents a serious challenge to extend the Theory. This article reviews both these aspects of flavour physics from a theoretical perspective.
\end{abstract}

\section{Introduction}

The term flavour is used, in the jargon of particle physics, to characterize the different copies of fields with the same spin and gauge quantum numbers, and flavour physics refers to the study of the interactions that distinguish between these copies. Within the Standard Theory (ST) of fundamental interactions, as we know it now, all matter fields (quark, leptons, and neutrinos) appear in three flavours, and the only interaction that distinguish these three flavours is the Yukawa interaction, or the interaction of the matter fields with the Higgs boson.

The fact that flavour non-universality is generated only by Yukawa interaction is an unavoidable consequence in the Standard Theory, given its particle content. However, this structure was far from being obvious for decades: from the discovery of strange particles in the 1950's till the triumph of the ST predictions for quarkflavour mixing observed at the $B$-factories in the 2000's. During all these years the progress in understanding flavour physics has been intimately related to the overall progress in building and testing the ST of fundamental interactions.

At present we have a clear understanding of the underlying mechanism of flavour mixing and flavour non-universality within the ST, and this mechanism has been successfully verified in experiments. However, flavour physics still represents one of the most puzzling and, at the same time, interesting aspects of particle physics. Our "ignorance" in this sector can be summarized by the following two open questions: 
- What determines the observed pattern of masses and mixing angles of quarks and leptons?

- Which are the sources of flavour symmetry breaking accessible at low energies? Is there anything else beside the ST Yukawa couplings?

Answering these questions is a key part of the more general program of investigating the nature of physics beyond the ST. There are indeed convincing arguments, including the peculiar pattern of quark and lepton masses, which motivate us to consider the ST as the low-energy limit of a more complete theory.

The precise understanding of the mechanism of flavour mixing within the ST, summarized in section 2-3, is essential to formulate the above questions in a quantitative way. The present status of the partial answers obtained so far to the second question, and their implications for physics beyond the ST, are presented in section 4-6. Some of the theoretical ideas put forwards to address the first question are presented in section 7 .

\section{Some historical remarks}

The first building block of what we now call flavour physics was laid down by Cabibbo in $1963,{ }^{1}$ well before many of the ingredients of the Standard Theory were clear. The Cabibbo theory of semileptonic decays provided the first step toward a unified description of hadronic and leptonic weak interactions. Later on, the hypothesis of the existence of the charm quark, formulated by Glashow, Iliopoulos and Maiani, ${ }^{2}$ represented a key ingredient both to understand the mechanism of quark flavour mixing within the ST and, at the same time, to understand how to extend the unified mechanism of weak and electromagnetic interactions from the lepton sector to the quark sector. Finally, the hypothesis formulated by Kobayashi and Maskawa ${ }^{3}$ that quarks appear in three flavour turned out to be the correct explanation of the phenomenon of CP violation within the ST.

The theoretical foundations of the mechanism of flavour mixing within the ST were anticipated and followed by a long series of key experimental observations, starting from the discovery of CP violation in the neutral kaon system in $1964,{ }^{4}$ and culminated with the precise determination of all the elements of the CabibboKobayashi-Maskawa quark-flavour mixing matrix at the $B$-meson factories, ${ }^{5}$ and at various dedicated $K$-decay experiments. ${ }^{6,7}$ At the completion of the $B$-factory program, it has became clear that the ST provides a successful description of the mechanism of quark flavour mixing: possible contributions due to New Physics (NP), if any, can only be small corrections compared to the leading ST terms. The search for such tiny deviations is the main goal of present and future experimental efforts in flavour physics. ${ }^{8-11}$

The precise comparison between data and ST in flavour physics has been made possible by a significant amount of theoretical progress in understanding how QCD interactions modify weak interactions at low energies. This started with the pioneer- 
ing work of Gaillard and Lee, ${ }^{12}$ and Altarelli and Maiani, ${ }^{13}$ further extended by Shifman, Vainshtein, and Zakharov, ${ }^{14}$ and by Gilman and M. B. Wise. ${ }^{15}$ A significant step forward was undertaken in the 1990's, where all the relevant flavour-changing processes have been computed at the next-to-leading order (NLO) accuracy, in particular by the Munich ${ }^{16}$ and Rome ${ }^{17}$ groups (see Ref. 18 for a complete list of NLO references). More recently specific processes, such as $B \rightarrow X_{s} \gamma$ and $B_{s, d} \rightarrow \ell^{+} \ell^{-}$, have been computed even at NNLO accuracy. ${ }^{19-21}$

\section{The flavour sector of the Standard Theory}

The ST Lagrangian can be divided into two main parts, the gauge and the Higgs (or symmetry breaking) sector. The gauge sector is extremely simple and highly symmetric: it is completely specified by the local symmetry $S U(3)_{C} \times S U(2)_{L} \times$ $U(1)_{Y}$ and by the fermion content. This consists of five fields with different quantum numbers under the gauge group: the $S U(2)_{L}$ doublet of quarks $\left(Q_{L}^{i}\right)$, the two righthanded quark singlets $\left(U_{R}^{i}\right.$ and $\left.D_{R}^{i}\right)$, the lepton doublet $\left(Q_{L}^{i}\right)$, and the right-handed lepton singlet $\left(E_{R}^{i}\right)$.

Each of these five different fields appears in three different replica or flavours $(i=1,2,3)$, giving rise to a large global flavour symmetry. Both the local and the global symmetries of the gauge sector of the ST are broken by the Higgs field. The local symmetry is spontaneously broken by the vacuum expectation value of the Higgs field, $\langle|\phi|\rangle=v=\left(2 \sqrt{2} G_{F}\right)^{-1 / 2} \approx 174 \mathrm{GeV}$, while the global flavour symmetry is explicitly broken by the Yukawa interaction of $\phi$ with the fermion fields:

$$
-\mathcal{L}_{\text {Yukawa }}^{\mathrm{ST}}=Y_{d}^{i j} \bar{Q}_{L}^{i} \phi D_{R}^{j}+Y_{u}^{i j} \bar{Q}_{L}^{i} \tilde{\phi} U_{R}^{j}+Y_{e}^{i j} \bar{L}_{L}^{i} \phi E_{R}^{j}+\text { h.c. } \quad\left(\tilde{\phi}=i \tau_{2} \phi^{\dagger}\right) .
$$

The large global flavour symmetry of $\mathcal{L}_{\text {gauge }}^{\mathrm{ST}}$, corresponding to the independent unitary rotations in flavour space of the five fermion fields, is a $U(3)^{5}$ group. $^{22}$ This can be decomposed as follows: $\mathcal{G}_{\text {flavour }}=U(1)^{5} \times \mathcal{G}_{q} \times \mathcal{G}_{\ell}$, where

$$
\mathcal{G}_{q}=S U(3)_{Q_{L}} \times S U(3)_{U_{R}} \times S U(3)_{D_{R}}, \quad \mathcal{G}_{\ell}=S U(3)_{L_{L}} \times S U(3)_{E_{R}} .
$$

Three of the five $U(1)$ subgroups can be identified with the total barion and lepton number, which are not broken by the Yukawa interaction, and the weak hypercharge, which is gauged and broken only spontaneously by $\langle\phi\rangle \neq 0$. The subgroups controlling flavour-changing dynamics and flavour non-universality are the non-Abelian groups $\mathcal{G}_{q}$ and $\mathcal{G}_{\ell}$, which are explicitly broken by $Y_{d, u, e}$ not being proportional to the identity matrix.

The diagonalization of each Yukawa matrix requires, in general, two independent unitary matrices, $V_{L} Y V_{R}^{\dagger}=\operatorname{diag}\left(y_{1}, y_{2}, y_{3}\right)$. In the lepton sector we are free to choose the two matrices necessary to diagonalize $Y_{e}$ without breaking gauge invariance. This is not the case in the quark sector, where we cannot diagonalize on the left both $Y_{d}$ and $Y_{u}$ at the same time. We are thus left with a non-trivial misalignment matrix $V$, between $Y_{d}$ and $Y_{u}$, which is nothing but the Cabibbo- 
Kobayashi-Maskawa (CKM) mixing matrix: ${ }^{1,3}$

$$
V=\left(\begin{array}{ccc}
V_{u d} & V_{u s} & V_{u b} \\
V_{c d} & V_{c s} & V_{c b} \\
V_{t d} & V_{t s} & V_{t b}
\end{array}\right)
$$

For practical purposes it is often convenient to work in the mass eigenstate basis of both up- and down-type quarks. This can be achieved rotating independently the up and down components of the quark doublet $Q_{L}$, or moving the CKM matrix from the Yukawa sector to the charged weak current in $\mathcal{L}_{\text {gauge }}^{\mathrm{ST}}$ :

$$
\left.J_{W}^{\mu}\right|_{\text {quarks }}=\bar{u}_{L}^{i} \gamma^{\mu} d_{L}^{i} \quad \stackrel{u, d \text { mass-basis }}{\longrightarrow} \bar{u}_{L}^{i} V_{i j} \gamma^{\mu} d_{L}^{j}
$$

However, it must be stressed that $V$ originates from the Yukawa sector (in particular by the miss-alignment of $Y_{u}$ and $Y_{d}$ in the $S U(3)_{Q_{L}}$ subgroup of $\mathcal{G}_{q}$ ): in the absence of Yukawa couplings we can always set $V_{i j}=\delta_{i j}$.

To summarize, quark flavour physics within the ST is characterized by a large flavour symmetry, $\mathcal{G}_{q}$, defined by the gauge sector, whose only breaking sources are the two Yukawa couplings $Y_{d}$ and $Y_{u}$. The CKM matrix arises by the miss-alignment of $Y_{u}$ and $Y_{d}$ in flavour space.

\subsection{The CKM matrix}

The residual invariance under the flavour group allows us to eliminate five of the six complex phases in $V$, that contains only four real physical parameters: three mixing angles and one CP-violating phase. The off-diagonal elements of the CKM matrix show a strongly hierarchical pattern: $\left|V_{u s}\right|$ and $\left|V_{c d}\right|$ are close to 0.22 , the elements $\left|V_{c b}\right|$ and $\left|V_{t s}\right|$ are of order $4 \times 10^{-2}$ whereas $\left|V_{u b}\right|$ and $\left|V_{t d}\right|$ are of $O\left(10^{-3}\right)$.

The Wolfenstein parametrization, namely the expansion of the CKM matrix elements in powers of the small parameter $\lambda \doteq\left|V_{u s}\right| \approx 0.22$, is a convenient way to exhibit this hierarchy in a more explicit way: ${ }^{23}$

$$
V=\left(\begin{array}{ccc}
1-\frac{\lambda^{2}}{2} & \lambda & A \lambda^{3}(\varrho-i \eta) \\
-\lambda & 1-\frac{\lambda^{2}}{2} & A \lambda^{2} \\
A \lambda^{3}(1-\varrho-i \eta) & -A \lambda^{2} & 1
\end{array}\right)+\mathcal{O}\left(\lambda^{4}\right) .
$$

Here $A, \varrho$, and $\eta$ are three independent parameters of order 1 . Because of the smallness of $\lambda$ and the fact that for each element the expansion parameter is actually $\lambda^{2}$, this is a rapidly converging expansion.

The unitarity of the CKM matrix implies a series of relations of the type $\sum_{k=1 \ldots 3} V_{k i}^{*} V_{k j}=\delta_{i j}$. These relations are a distinctive feature of the ST, where the CKM matrix is the only source of quark flavour mixing. Their experimental verification is therefore a useful tool to set bounds on, or possibly reveal, new sources of flavour symmetry breaking. Among these relations, the one obtained for $i=1$ and $j=3$, namely

$$
V_{u d} V_{u b}^{*}+V_{c d} V_{c b}^{*}+V_{t d} V_{t b}^{*}=0
$$



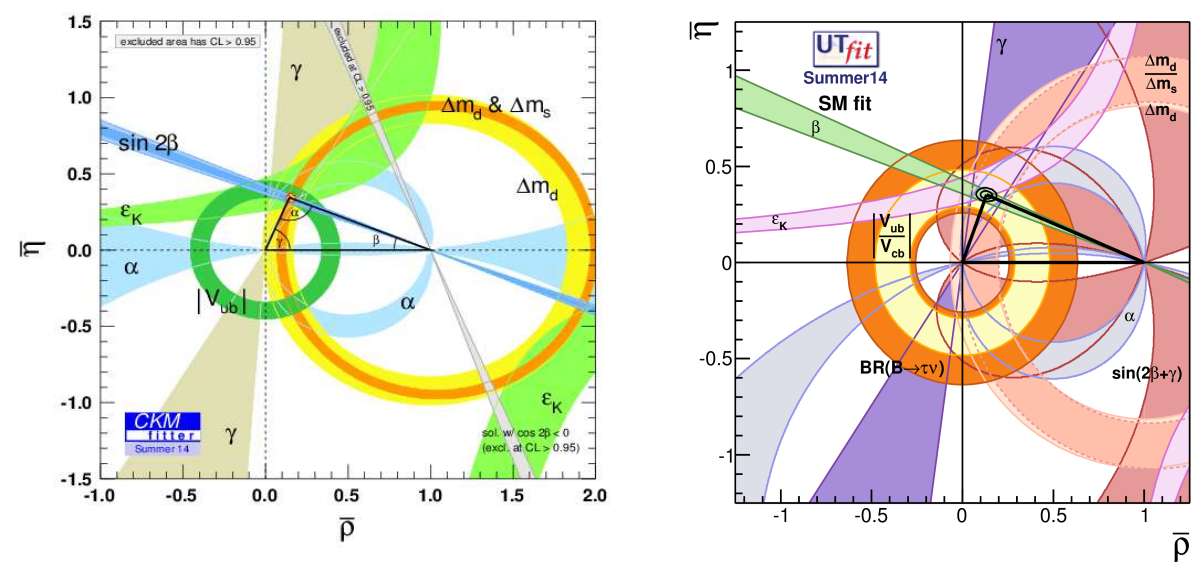

Fig. 1. Allowed region in the $\bar{\varrho}, \bar{\eta}$ plane as obtained by the CKMfitter ${ }^{24}$ and $\mathrm{UTfit}^{25}$ collabora- $^{2}$ tions. Superimposed are the individual constraints from charmless semileptonic $B$ decays $\left(\left|V_{u b}\right|\right)$, mass differences in the $B_{d}\left(\Delta m_{d}\right)$ and $B_{s}\left(\Delta m_{s}\right)$ systems, CP violation in the neutral kaon $\left(\varepsilon_{K}\right)$ and in the $B_{d}$ systems $(\sin 2 \beta)$, the combined constrains on $\alpha$ and $\gamma$ from various $B$ decays.

$$
\text { or } \quad \frac{V_{u d} V_{u b}^{*}}{V_{c d} V_{c b}^{*}}+\frac{V_{t d} V_{t b}^{*}}{V_{c d} V_{c b}^{*}}+1=0 \quad \leftrightarrow \quad[\rho+i \eta]+[(1-\rho)-i \eta]+1=0,
$$

is particularly interesting since it involves the sum of three terms all of the same order in $\lambda$ and is usually represented as a unitarity triangle in the complex plane (see Fig. 1). We stress that Eq. (6) is invariant under any phase transformation of the quark fields. Under such transformations the unitarity triangle is rotated in the complex plane, but its angles and the sides remain unchanged. Both angles and sides of the unitary triangle are indeed observable quantities which can be measured in suitable experiments.

The values of $\left|V_{u s}\right|$ and $\left|V_{c b}\right|$ (or $\lambda$ and $A$ ), are determined with good accuracy from $K \rightarrow \pi \ell \nu$ and $B \rightarrow X_{c} \ell \nu$ decays, respectively. Using these inputs, all the other constraints on the elements of the CKM matrix can be expressed as constraints on $\rho$ and $\eta$. The list of the most sensitive observables used to (over) determine the CKM matrix elements include (see Fig. 1):

- The rates of inclusive and exclusive charmless semileptonic $B$ decays, that depend on $\left|V_{u b}\right|$.

- The phase of the $B_{d}-\bar{B}_{d}$ mixing amplitude (measured from the timedependent $\mathrm{CP}$ asymmetry in $B \rightarrow \psi K_{S}$ decays), that depends on $\sin 2 \beta$.

- The rates of various $B \rightarrow D K$ decays constraining the angle $\gamma$.

- The rates of various $B \rightarrow \pi \pi, \rho \pi, \rho \rho$ decays constraining the combination $\alpha=\pi-\beta-\gamma$.

- The ratio between the mass splittings in the neutral $B$ and $B_{s}$ systems, that depends on $\left|V_{t d} / V_{t s}\right|$. 
- The indirect $\mathrm{CP}$ violating parameter of the kaon system $\left(\epsilon_{K}\right)$, that determines a hyperbola in the $\rho-\eta$ plane.

The resulting constraints, as implemented by the CKMfitter and UTfit collaborations, are shown in Fig. 1. As can be seen, they are all consistent with a unique value of $\bar{\varrho}=\rho\left(1-\frac{\lambda^{2}}{2}\right)$ and $\bar{\eta}=\eta\left(1-\frac{\lambda^{2}}{2}\right)$.

The consistency of different constraints on the CKM unitarity triangle is a powerful consistency test of the ST in describing flavour-changing phenomena. From the plot in Fig. 1 it is quite clear, at least in a qualitative way, that there is little room for non-ST contributions in flavour changing transitions. A more quantitative evaluation of this statement is presented in the next section.

\section{The flavour problem}

As anticipated in the introduction, despite the impressive phenomenological success of the ST, there are various convincing arguments which motivate us to consider this model only as the low-energy limit of a more complete theory.

Assuming that the new degrees of freedom which complete the theory are heavier than the ST particles, we can integrate them out and describe physics beyond the ST in full generality by means of an effective field theory (EFT) approach. The ST Lagrangian becomes the renormalizable part of a more general local Lagrangian which includes an infinite tower of operators with dimension $d>4$, constructed in terms of the ST fields and suppressed by inverse powers of an effective scale $\Lambda$. These operators are the residual effect of having integrated out the new heavy degrees of freedom, whose mass scale is parametrized by the effective scale $\Lambda>m_{W}$.

Integrating out heavy degrees of freedom is a procedure often adopted also within the ST: it allows us to simplify the evaluation of amplitudes which involve different energy scales. This approach is indeed a generalization of the Fermi theory of weak interactions, where the dimension-six four-fermion operators describing weak decays are the results of having integrated out the $W$ field. The only difference when applying this procedure to physics beyond the ST is that in this case, as also in the original work by Fermi, we don't know the nature of the degrees of freedom we are integrating out. This implies we are not able to determine a priori the values of the effective couplings of the higher-dimensional operators. The advantage of this approach is that it allows us to analyse all realistic extensions of the ST in terms of a limited number of free parameters.

The Lagrangian of the ST considered as an effective theory can be written as follows

$$
\mathcal{L}_{\text {eff }}=\mathcal{L}_{\text {gauge }}^{\mathrm{ST}}+\mathcal{L}_{\text {Higgs }}^{\mathrm{ST}}+\mathcal{L}_{\text {Yukawa }}^{\mathrm{ST}}+\Delta \mathcal{L}_{d>4}
$$

where $\Delta \mathcal{L}_{d>4}$ denotes the series of higher-dimensional operators invariant under the ST gauge group. The coefficients of these operators have the form $c_{i} / \Lambda^{\left(d_{i}-4\right)}$, where $c_{i}$ is an adimensional coefficient and $d_{i}$ denotes the canonical dimension of 

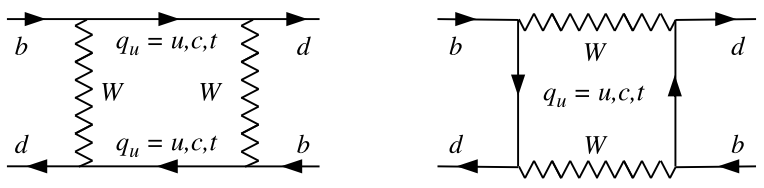

Fig. 2. Box diagrams contributing to $B_{d}-\bar{B}_{d}$ mixing in the unitary gauge.

the effective operator. If the new dynamics appears at the $\mathrm{TeV}$ scale, as we expect from a natural stabilization of the mechanism of electroweak symmetry breaking, the scale $\Lambda$ cannot exceed a few TeV. Moreover, from naturalness arguments, ${ }^{26}$ we should also expect that all the adimensional coefficients $c_{i}$ are of $O(1)$ unless suppressed by some symmetry argument. The observation that this expectation is not fulfilled by several dimension-six operators contributing to flavour-changing processes is often denoted as the flavour problem.

If the ST Lagrangian were invariant under some flavour symmetry, this problem could easily be circumvented. For instance in the case of baryon- or lepton-number violating processes, which are exact symmetries of the ST Lagrangian, we can avoid the tight experimental bounds promoting $B$ and $L$ to be exact symmetries of the new dynamics at the $\mathrm{TeV}$ scale. The peculiar aspects of flavour physics is that there is no exact flavour symmetry in the low-energy theory. In this case it is not sufficient to invoke a flavour symmetry for the underlying dynamics. We also need to specify how this symmetry is broken in order to describe the observed low-energy spectrum and, at the same time, be in agreement with the precise experimental tests of flavour-changing processes.

The best way to quantify the flavour problem is obtained by looking at consistency of the tree- and loop-mediated constraints on the CKM matrix. In first approximation we can assume that New Physics (NP) effects are negligible in processes which are dominated by tree-level amplitudes. Following this assumption, the values of $\left|V_{u s}\right|,\left|V_{c b}\right|$, and $\left|V_{u b}\right|$, as well as the constraints on $\alpha$ and $\gamma$ can be considered as NP free. As can be seen in Fig. 1, this implies we can determine completely the CKM matrix assuming generic NP effects in loop-mediated amplitudes. We can then use the measurements of observables which are loop-mediated within the ST to bound the couplings of effective higher-dimensional operators which contribute to these observables at the tree level.

The loop-mediated constraints shown in Fig. 1 are those from the mixing of $B_{d}$, $B_{s}$, and $K^{0}$ with the corresponding anti-particles (generically denoted as $\Delta F=2$ amplitudes). Within the ST, these processes are generated by box amplitudes of the type in Fig. 2 (and similarly for $B_{s}$, and $K^{0}$ ) and are affected by small hadronic uncertainties. The leading contribution is obtained with the top-quark running inside the loop, giving rise to the highly suppressed result

$$
\mathcal{M}_{\Delta F=2}^{\mathrm{ST}} \approx \frac{G_{F}^{2} m_{t}^{2}}{16 \pi^{2}} V_{3 i}^{*} V_{3 j}\left\langle\bar{M}\left|\left(\bar{d}_{L}^{i} \gamma^{\mu} d_{L}^{j}\right)^{2}\right| M\right\rangle \times F\left(\frac{m_{t}^{2}}{m_{W}^{2}}\right) \quad\left[M=K^{0}, B_{d}, B_{s}\right],
$$




\begin{tabular}{c|cc|cc|c}
\hline \hline Operator & \multicolumn{2}{|c|}{$\Lambda$ in TeV $\left(c_{\mathrm{NP}}=1\right)$} & \multicolumn{2}{|c|}{ Bounds on $c_{\mathrm{NP}}(\Lambda=1 \mathrm{TeV})$} & \multirow{2}{*}{ Observables } \\
& $\mathrm{Re}$ & $\mathrm{Im}$ & $\mathrm{Re}$ & $\operatorname{Im}$ & \\
\hline$\left(\bar{s}_{L} \gamma^{\mu} d_{L}\right)^{2}$ & $9.8 \times 10^{2}$ & $1.6 \times 10^{4}$ & $9.0 \times 10^{-7}$ & $3.4 \times 10^{-9}$ & $\Delta m_{K} ; \epsilon_{K}$ \\
$\left(\bar{s}_{R} d_{L}\right)\left(\bar{s}_{L} d_{R}\right)$ & $1.8 \times 10^{4}$ & $3.2 \times 10^{5}$ & $6.9 \times 10^{-9}$ & $2.6 \times 10^{-11}$ & $\Delta m_{K} ; \epsilon_{K}$ \\
\hline$\left(\bar{c}_{L} \gamma^{\mu} u_{L}\right)^{2}$ & $1.2 \times 10^{3}$ & $2.9 \times 10^{3}$ & $5.6 \times 10^{-7}$ & $1.0 \times 10^{-7}$ & $\Delta m_{D} ;|q / p|, \phi_{D}$ \\
$\left(\bar{c}_{R} u_{L}\right)\left(\bar{c}_{L} u_{R}\right)$ & $6.2 \times 10^{3}$ & $1.5 \times 10^{4}$ & $5.7 \times 10^{-8}$ & $1.1 \times 10^{-8}$ & $\Delta m_{D} ;|q / p|, \phi_{D}$ \\
\hline$\left(b_{L} \gamma^{\mu} d_{L}\right)^{2}$ & $6.6 \times 10^{2}$ & $9.3 \times 10^{2}$ & $2.3 \times 10^{-6}$ & $1.1 \times 10^{-6}$ & $\Delta m_{B_{d}} ; S_{\psi K_{S}}$ \\
$\left(\bar{b}_{R} d_{L}\right)\left(\bar{b}_{L} d_{R}\right)$ & $2.5 \times 10^{3}$ & $3.6 \times 10^{3}$ & $3.9 \times 10^{-7}$ & $1.9 \times 10^{-7}$ & $\Delta m_{B_{d}} ; S_{\psi K_{S}}$ \\
\hline$\left(\bar{b}_{L} \gamma^{\mu} s_{L}\right)^{2}$ & $1.4 \times 10^{2}$ & $2.5 \times 10^{2}$ & $5.0 \times 10^{-5}$ & $1.7 \times 10^{-5}$ & $\Delta m_{B s} ; S_{\psi \phi}$ \\
$\left(\bar{b}_{R} s_{L}\right)\left(\bar{b}_{L} s_{R}\right)$ & $4.8 \times 10^{2}$ & $8.3 \times 10^{2}$ & $8.8 \times 10^{-6}$ & $2.9 \times 10^{-6}$ & $\Delta m_{B_{s}} ; S_{\psi \phi}$ \\
\hline \hline
\end{tabular}

Table 1: Bounds on representative dimension-six $\Delta F=2$ operators with effective coupling $c_{\mathrm{NP}} / \Lambda^{2}$. The bounds are quoted on $\Lambda$, setting $\left|c_{\mathrm{NP}}\right|=1$, or on $c_{\mathrm{NP}}$, setting $\Lambda=1 \mathrm{TeV}$. The right column denotes the main observables used to derive these bounds. ${ }^{27}$

where $F$ is a loop function of $O(1)$ and $i, j$ denote the flavour indexes of the meson valence quarks.

Magnitude and phase of all these mixing amplitudes have been determined with good accuracy from experiments and are consistent with the ST predictions. To translate this information into bounds on the scale of new physics, let's consider the following set of $\Delta F=2$ dimension-six operators in $\Delta \mathcal{L}_{d>4}$ :

$$
\Delta \mathcal{L}_{d>4} \supset \sum \frac{c_{i j}}{\Lambda^{2}} \mathcal{O}_{\Delta F=2}^{i j} \quad \mathcal{O}_{\Delta F=2}^{i j}=\left(\bar{q}_{L}^{i} \gamma^{\mu} q_{L}^{j}\right)^{2}
$$

These operators contribute at the tree-level to the meson-antimeson mixing amplitudes. The condition $\left|\mathcal{M}_{\Delta F=2}^{\mathrm{NP}}\right|<\left|\mathcal{M}_{\Delta F=2}^{\mathrm{ST}}\right|$ implies

$$
\Lambda<\frac{3.4 \mathrm{TeV}}{\left|V_{3 i}^{*} V_{3 j}\right| /\left|c_{i j}\right|^{1 / 2}}<\left\{\begin{array}{lll}
9 \times 10^{3} \mathrm{TeV} \times\left|c_{21}\right|^{1 / 2} & \text { from } & K^{0}-\bar{K}^{0} \\
4 \times 10^{2} \mathrm{TeV} \times\left|c_{31}\right|^{1 / 2} & \text { from } & B_{d}-\bar{B}_{d} \\
7 \times 10^{1} \mathrm{TeV} \times\left|c_{32}\right|^{1 / 2} & \text { from } & B_{s}-\bar{B}_{s}
\end{array}\right.
$$

A more refined analysis, with complete statistical treatment and separate bounds for the real and the imaginary parts of the various amplitudes, considering also operators with different Dirac structure, leads to the bounds reported in Table 1. The main message of these bounds is the following:

- New physics models with a generic flavour structure $\left(c_{i j}\right.$ of order 1$)$ at the $\mathrm{TeV}$ scale are ruled out. If we want to keep $\Lambda$ in the $\mathrm{TeV}$ range, physics beyond the ST must have a highly non-generic flavour structure.

In the specific case of the $\Delta F=2$ operators in (9), in order to keep $\Lambda$ in the TeV range, we must find a symmetry argument such that $\left|c_{i j}\right| \lesssim\left|V_{3 i}^{*} V_{3 j}\right|^{2}$. Reproducing a similar structure beyond the ST is a highly non-trivial task. However, as discussed below, it can be obtained under specific assumptions. 


\section{The Minimal Flavour Violation hypothesis}

The "protection" of $\Delta F=2$ observables and, more generally, flavour-changing neutral-current (FCNC) processes occurring within the ST is a consequences of the specific symmetry and symmetry-breaking structure of the ST Lagrangian discussed in section 3. In particular, the fact that the quark flavour group $\mathcal{G}_{q}$ is broken only by the two quark Yukawa couplings, and that the top-quark Yukawa coupling is the only $O(1)$ entry in $Y_{u, d}$, is the main reason why Eq. (8) is highly suppressed.

The strongest assumption we can make to suppress flavour-changing effects in generic extensions of the ST is the so-called Minimal Flavour Violation (MFV) hypothesis, namely the assumption that $Y_{u}$ and $Y_{d}$ are the only sources of flavour symmetry breaking also beyond the ST. ${ }^{22,28,29}$ To implement and interpret this hypothesis in a consistent way, we can assume that $\mathcal{G}_{q}$ is a good symmetry and promote $Y_{u, d}$ to be non-dynamical fields (spurions) with non-trivial transformation properties under $\mathcal{G}_{q}$ :

$$
Y_{u} \sim(3, \overline{3}, 1), \quad Y_{d} \sim(3,1, \overline{3}) .
$$

Employing the EFT language, an effective theory satisfies the MFV criterion in the quark sector if all higher-dimensional operators, constructed from ST fields and the $Y_{u, d}$ spurions, are formally invariant under the flavour group $\mathcal{G}_{q} \cdot{ }^{29}$ The dynamical idea behind this construction is the hypothesis that the breaking of the symmetry occurs at very high energy scales, and that $Y_{u, d}$ are the only independent combination of breaking terms (e.g. combination of appropriate vacuum expectation values) that survive at low energies.

According to the MFV criterion one should in principle consider operators with arbitrary powers of the (dimensionless) Yukawa fields. However, a strong simplification arises by the observation that all the eigenvalues of the Yukawa matrices are small, but for the top-quark one, and that the off-diagonal elements of the CKM matrix are very suppressed. This fact is enough to ensure that, even when including high powers of $Y_{u}$ and $Y_{d}$, FCNC amplitudes get exactly the same CKM suppression as in the ST:

$$
\mathcal{M}_{\Delta F=1}^{\mathrm{MFV}}\left(d^{i} \rightarrow d^{j}\right) \propto\left(V_{t i}^{*} V_{t j}\right), \quad \mathcal{M}_{\Delta F=2}^{\mathrm{MFV}}\left(d^{i} \bar{d}^{j} \rightarrow d^{j} \bar{d}^{i}\right) \propto\left(V_{t i}^{*} V_{t j}\right)^{2} .
$$

The proportionality constants in these relations are flavour universal, implying the same NP correction (relative to the ST) in $s \rightarrow d, b \rightarrow d$, and $b \rightarrow s$ transitions.

As a consequence of this structure, within the MFV framework several of the constraints used to determine the CKM matrix (and in particularly the unitarity triangle in Fig. 1) are not affected by NP. ${ }^{30}$ For instance, the structure of the basic flavour-changing coupling in Eq. (12) implies that the weak CPV phase of $B_{d^{-}}$ $\bar{B}_{d}$ mixing is $\arg \left[\left(V_{t d} V_{t b}^{*}\right)^{2}\right]$, exactly as in the ST. This construction thus provides a natural (a posteriori) justification of why no NP effects have been observed in the quark sector. Moreover, the built-in CKM suppression leads to bounds on the 
effective scale of new physics in the few $\mathrm{TeV}$ domain . These bounds are very similar to the bounds on flavour-conserving operators derived by precision electroweak tests.

A few additional comments about the MFV hypothesis are listed below:

- Although MFV seems to be a natural solution to the flavour problem, we are still far from having proved the validity of this hypothesis from data. A proof of the MFV hypothesis can be achieved only with a positive evidence of NP exhibiting the flavour-universality pattern predicted by MFV (same relative correction in $s \rightarrow d, b \rightarrow d$, and $b \rightarrow s$ transitions of the same type). This could happens, for instance, via precise measurements of the rare decays $B_{s} \rightarrow \mu^{+} \mu^{-}$and $B_{d} \rightarrow \mu^{+} \mu^{-} .31-33$ Conversely, an evidence of NP in flavour-changing transitions not respecting the MFV pattern (e.g. an evidence of $\mathcal{B}\left(B_{d} \rightarrow \mu^{+} \mu^{-}\right)$well above its ST prediction) would not only imply the existence of physics beyond the ST, but also the existence of new sources of flavour symmetry breaking beyond the Yukawa couplings.

- The MFV ansatz is quite successful on the phenomenological side; however, it is unlikely to be an exact property of the model valid to all energy scales. Despite some recent attempts to provide a dynamical justification of this symmetry-breaking ansatz, the most natural possibility is that MFV is only an accidental low-energy property of the theory. It could also well be that a less minimal connection between NP flavour-violating couplings and Yukawa couplings is at work. It is then very important to search for possible deviations (even if tiny) from the MFV predictions.

- Even if the MFV ansatz holds, it does not necessarily imply small deviations from the ST predictions in all flavour-changing phenomena. The MFV ansatz can be implemented in different ways. For instance, in models with two Higgs doublets we can change the relative normalization of the two Yukawa couplings. ${ }^{29}$ It is also possible to decouple the breaking of $\mathrm{CP}$ invariance from the breaking of the $\mathcal{G}_{q}$ quark-flavour group, ${ }^{34}$ leaving more room for NP in CP-violating observables. All these variations lead to different and well defined patterns of possible deviations from the ST that we have only started to investigate and that represent one of the main goal of present and future experiments in flavour physics. ${ }^{8-11}$

- The usefulness of the MFV ansatz is closely linked to the theoretical expectation of $\mathrm{NP}$ in the $\mathrm{TeV}$ range. This expectation follows from a natural stabilization of the Higgs sector, but it is in tension with the lack of any direct signal of NP at the LHC. The more the scale of NP is pushed up, the more it is possible to allow sizable deviations from the MFV ansatz.

\section{Flavour symmetry breaking beyond MFV}

As anticipated, MFV is not the only option to "protect" flavour-changing transitions in extensions of the ST. A key feature common to most models able to accommodate 
NP not far from the TeV scale, ensuring a sufficient suppression of flavour-changing transitions, is some link between flavour-changing amplitudes and fermion masses. Indeed the strong phenomenological bounds on flavour-changing transitions always involve light quarks (or leptons) of the first two generations, and are particularly strong in the case of transitions among the first two families (see Table 1). Given the smallness of fermion masses of the first two generations, a link between flavourchanging amplitudes and fermion masses provides a good starting point for a natural suppression of flavour-changing transitions.

In the quark sector this link can be efficiently implemented considering only the $U(2)^{3}$ subgroup of the full quark flavour group $\left(\mathcal{G}_{q}\right)$ that is obtained in the limit of vanishing Yukawa couplings for the first two generations of quarks. ${ }^{34,35}$ This symmetry limit is a better approximation of the full ST Lagrangian, since top and bottom quarks are allowed to have a non-vanishing mass. The $U(2)^{3}$ subgroup is also sufficient to ensure enough protection from flavour-changing transitions beyond the ST, assuming the minimal breaking structure necessary to describe light fermion masses. The main difference of this ansatz compared to the MFV hypothesis is the breaking of the universal link between $s \rightarrow d$ transitions vs. transitions involving third generation quarks $(b \rightarrow d$ and $b \rightarrow s$ ).

So far we discussed mainly the quark sector, but a flavour problem exists also in the lepton sector. Similarly to the $\Delta F=2$ bounds in Table 1, the strong experimental bounds on FCNC transitions of charged leptons $(\mu \rightarrow e \gamma, \mu \rightarrow 3 e$, $\mu N \rightarrow e N, \tau \rightarrow \mu \gamma, \ldots)$ can be translated into bounds on NP scales well above the $\mathrm{TeV}$, for $O(1)$ flavour-changing coefficients. For instance the MEG bound ${ }^{36}$ $\mathcal{B}(\mu \rightarrow e \gamma)<5.7 \times 10^{-13}$ leads to an effective bound on $\Lambda$ of the order of $10^{5} \mathrm{TeV}$.

In order to allow $\mathrm{TeV}$ scale NP, some extension of the MFV hypothesis can be implemented also is the lepton sector. However, given there is not a unique way to accommodate non-vanishing neutrino masses, in this case there is more freedom to define the minimal sources of flavour symmetry breaking. Different versions of Minimal Lepton Flavour Violation (MLFV) have been proposed in the literature, depending on how the irreducible breaking terms in the neutrino sector are identifed. ${ }^{37-41}$ On general grounds, it is not difficult to provide a sufficient suppression of flavour-changing coefficients for $\mathrm{TeV}$ scale new physics, provided the (adimensional) flavour breaking terms associated to neutrino masses are sufficiently small. In the context of see-saw models, this imply masses for the heavy righthanded neutrinos typically around or below $10^{12} \mathrm{GeV} \cdot{ }^{37}$ A significant progress in this field is expected by the next generation of LFV experiments with charged leptons, especially in the sector of $\mu \rightarrow e$ transitions. ${ }^{42}$ As for the quark sector, the key tool to test flavour symmetries (and symmetry-breaking) assumptions relies on the observation of possible correlations in the rate of neutral-current LFV processes, such as $\tau \rightarrow \mu \gamma$ vs. $\mu \rightarrow e \gamma$. 


\section{Flavor physics and partial compositeness}

In the previous two sections we have discussed mechanisms to suppress flavourchanging transitions beyond the ST due to specific flavour symmetries and symmetry-breaking patterns. An interesting alternative is the possibility of a generic dynamical suppression of flavour-changing interactions, related to the weak mixing of the light ST fermions with some new dynamics occurring at the TeV scale. This is what happens in the so-called models with partial compositeness, ${ }^{43,44}$ where the hierarchy of fermion masses is attributed to the hierarchical mixing of the ST fermions with the heavier (composite) states of the theory.

Also the general features of this class of models can be described by means of an effective theory approach. ${ }^{45,46}$ The two main assumptions of this EFT approach are the following:

- There exists a (non-canonical) basis for the ST fermions where their kinetic terms exhibit a rather hierarchical form:

$$
\begin{aligned}
& \mathcal{L}_{\text {kin }}^{\text {quarks }}=\sum_{\Psi=Q_{L}, U_{R}, D_{R}} \bar{\Psi} Z_{\psi}^{-2} \not D \Psi, \\
& Z_{\psi}=\operatorname{diag}\left(z_{\psi}^{(1)}, z_{\psi}^{(2)}, z_{\psi}^{(3)}\right), \quad z_{\psi}^{(1)} \ll z_{\psi}^{(2)} \ll z_{\psi}^{(3)} \lesssim 1 .
\end{aligned}
$$

- In such basis there is no flavour symmetry and all the flavour-violating interactions, including the Yukawa couplings, are $\mathcal{O}(1)$.

Once the fields are transformed into the canonical basis, the hierarchical kinetic terms act as a distorting lens, through which all interactions are seen as approximately aligned on the magnification axes of the lens. The hierarchical $z_{\psi}^{(i)}$ can be interpreted as the effect of the mixing of an elementary (ST-like) sector of massless fermions with a corresponding set of heavy composite fermions: the elementary fermions feel the breaking of the electroweak (and flavour) symmetry only via this mixing.

The values of the $z_{\psi}^{(i)}$ can be deduced, up to an overall normalization, from the know structure of the Yukawa couplings, that can be decomposed as follows

$$
Y_{u}^{i j} \sim z_{Q}^{(i)} z_{U}^{(j)}, \quad Y_{d}^{i j} \sim z_{Q}^{(i)} z_{D}^{(j)} .
$$

Inverting such relations we can express the $z_{\psi}^{(i)}$ combinations appearing in the effective couplings of dimension-six operators involving ST fields [e.g. the combination $\left(z_{Q}^{(1)} z_{Q}^{(2)}\right)^{2}$ for the operator $\left(\bar{s}_{L} \gamma_{\mu} d_{L}\right)^{2}$, etc...] into appropriate powers of quark masses and CKM angles. The resulting suppression of FCNC amplitudes turns out to be quite effective being linked to the hierarchical structure of the Yukawa couplings.

As shown in a recent analysis, ${ }^{46}$ this framework is compatible with the strong flavour bounds in kaon sector for scales of the composite states (vector resonances) around $10 \mathrm{TeV}$. In this case one can expect deviations from the ST at the present level of experimental sensitivity in the electric dipole moment (EDM) of the neutron 
(where there is actually a significant tension with the present bound), CP-violating observables in the kaon system $\left(\epsilon^{\prime} / \epsilon\right.$ and $\left.\epsilon_{K}\right)$, and $b \rightarrow s$ FCNC transitions. However, in the lepton sector the minimal framework is not satisfactory (a severe finetuning is needed to satisfy current bounds on lepton-flavour violating processes).

It should be stressed that also in partial-compositeness models is possible to postulate the existence of additional protective flavour symmetries (as discussed e.g. in Ref. 48-50) and, for instance, recover a MFV structure. In this case the bounds on the composite states turn out to be well below $10 \mathrm{TeV}$.

\section{Dynamical Yukawa couplings}

The MFV principle does not provide an explanation for the observed pattern of masses and mixings of quarks and leptons: the Yukawa couplings are simply treated as inputs, as in the ST. To a large extent, also the mechanism of partial compositeness does not explain the observed pattern of quark and lepton Yukawa couplings: the hierarchal mixing between elementary and composite fermions is an input of the construction.

A more ambitious goal is that of deriving the observed structure of the Yukawa couplings from some fundamental principle. The simplest realization of the idea of a dynamical character for the Yukawa couplings is to assume that

$$
Y=\frac{\langle 0|\Phi| 0\rangle}{\Lambda}
$$

with $\Lambda$ being some high energy scale and $\Phi$ a set of scalar fields (or composite operators) with transformation properties such as to make invariant the effective potential $V(Y)$ under the flavour group $\mathcal{G}_{\text {flavour }}$ (or some of its subgroups). A general problem that one encounters along this line is the unwanted appearance of a large number of Goldstone bosons, associated to the spontaneous breaking of the large global continuos flavour symmetry. This problem could be avoided assuming that the flavour symmetry is gauged at some high energy scale. ${ }^{51}$

An interesting alternative to continuos flavour symmetries, that naturally avoids the problem of Goldstone bosons, is the possibility that the fundamental flavour symmetry is a suitable discrete subgroup of $\mathcal{G}_{\text {flavour }}$. This option has received a lot of attention in the recent past, mainly because of neutrino physics: ${ }^{52}$ the neutrino mixing matrix exhibits an almost tri-bimaximal structure and the latter is naturally expected in the context of discrete flavour symmetries. However, the description of both quark and lepton sectors in terms of a unique discrete flavour symmetry is less trivial and significantly more complicated. ${ }^{52-54}$ Moreover, this option has become less appealing also in the pure neutrino sector after the observation of a sizable 1-3 neutrino mixing angle, ${ }^{55,56}$ that implies sizable deviations from the tri-bimaximal mixing structure.

The idea that quark masses and, more generally, the Yukawa couplings, could arise from the minimization of a potential invariant under some continuos flavour 
symmetry is an old idea. Earlier attempts dates back to the sixties, when Michel and Radicati, ${ }^{57}$ and Cabibbo and Maiani ${ }^{58}$ developed generic group-theoretical methods to identify the natural extrema of $S U(3)_{L} \times S U(3)_{R}$ invariant potentials. Several further attempts towards a dynamical origin of the Yukawa couplings, employing various subgroups of $\mathcal{G}_{\text {flavour }}$ have been discussed in the literature. ${ }^{59-69}$ In models based on small symmetry groups, such as the $U(1)$ horizontal symmetry originally proposed by Froggat and Nielsen, ${ }^{59}$ it is quite easy to reproduce the observed mass matrices in terms of a reduced number of free parameters, while it is difficult to avoid problems with FCNCs, unless some amount of fine-tuning is introduced.

In models based on large (MFV-like) symmetry groups, it is difficult to explain the full pattern of quark and lepton masses in absence of significant fine-tuning among the coefficients of the potential. ${ }^{66}$ In this context, an interesting recent development has been presented in Ref. 70. There it has been shown that, among the most stable solution of the general minimization problem of $V(Y)$, corresponding to maximally unbroken subgroups ${ }^{57,58}$ of $\mathcal{G}_{\text {flavour }}$, there exists a class of solutions quite close to a realistic spectrum. In the quark sector, this corresponds to a hierarchical mass pattern of the third vs. the first two generations, with unity CKM matrix. In the lepton sector, it implies hierarchical masses for charged leptons and degenerate Majorana neutrinos, with one maximal, one large, and one vanishing mixing angle. Both these textures are close to the real situation, and can be brought in full agreement with data adding small perturbations. In the neutrino sector, this implies a firm prediction that can be tested in the near future, namely an almost degenerate spectrum with an average neutrino mass close to $m_{\nu} \approx 0.1 \mathrm{eV}$.

The radical alternative to predictions of quark and lepton masses based on continuos or discrete symmetries is the idea that they are simply random variables, possibly selected by anthropic arguments. The latter option has recently gained consensus, given the lack of deviations from the ST after the first run of the LHC. ${ }^{71}$ Drawing any firm conclusion in this respect is very difficult, and it will remain so also in the future. However, it is worth to stress that the measurement of the absolute value of neutrino masses could provide a significant additional piece of this fascinating puzzle: a value close to the present bounds, compatible with the hypothesis of a degenerate spectrum, would certainly speak in favour of some underlying large and mildly broken flavour symmetry. ${ }^{70,72}$

\section{Conclusions}

Flavour physics has a twofold role in investigating the nature of physics beyond the $\mathrm{ST}$. On the one hand, for NP models with new particles close to the $\mathrm{TeV}$ scale, existing low-energy flavour-physics measurements put very stringent limits on the flavour structure of the model. As illustrated in general terms and with a few specific examples, for such models present data tell us that the new degrees of freedom must have a highly non-generic flavour structure. However, this structure has not been clearly identified yet. In this perspective, if direct signals of NP will appear during 
the next LHC run, future progress in flavour physics will be an essential tool to investigate the peculiar flavour structure of the new degrees of freedom.

On the other hand, the paradigm of $\mathrm{NP}$ at the $\mathrm{TeV}$ scale is seriously challenged by the absence of deviations from the SM at the high-energy frontier. In this perspective, flavour physics remains a very powerful tool to search for physics beyond the ST, being potentially sensitive to NP scales much higher than those directly accessible at present and near-future high-energy facilities.

Acknowledgements I thank Admir Greljo, Luciano Maiani, and David Marzocca for useful comments on the manuscript. This work was supported in part by the Swiss National Science Foundation (SNF) under contract 200021-159720.

\section{References}

1. N. Cabibbo, Phys. Rev. Lett. 10, 531 (1963).

2. S. L. Glashow, J. Iliopoulos, L. Maiani, Phys. Rev. D 2 (1970) 1285.

3. M. Kobayashi, T. Maskawa, Prog. Theor. Phys. 49, 652 (1973).

4. J. H. Christenson, J. W. Cronin, V. L. Fitch, R. Turlay, Phys. Rev. Lett. 13 (1964) 138.

5. A. J. Bevan et al. [BaBar \& Belle Collab.], Eur. Phys. J. C 74 (2014) 11, 3026.

6. M. Antonelli et al. [FlaviaNet Kaon Working Group], Eur. Phys. J. C 69 (2010) 399.

7. V. Cirigliano, G. Ecker, H. Neufeld, A. Pich and J. Portoles, Rev. Mod. Phys. 84 (2012) 399.

8. R. Aaij et al. [LHCb Collaboration], Eur. Phys. J. C 73 (2013) 4, 2373.

9. T. Abe et al. [Belle-II Collaboration], arXiv:1011.0352.

10. M. Moulson [NA62 Collaboration], arXiv:1310.7816.

11. T. Yamanaka [KOTO Collaboration], PTEP 2012 (2012) $02 \mathrm{~B} 006$.

12. M. K. Gaillard, B. W. Lee, Phys. Rev. D 10 (1974) 897; Phys. Rev. Lett. 33 (1974) 108.

13. G. Altarelli, L. Maiani, Phys. Lett. B 52 (1974) 351.

14. M. A. Shifman, A. I. Vainshtein, V. I. Zakharov, Nucl. Phys. B 120 (1977) 316.

15. F. J. Gilman, M. B. Wise, Phys. Rev. D 20 (1979) 2392.

16. G. Buchalla, A. J. Buras, Nucl. Phys. B 400 (1993) 225.

17. M. Ciuchini, E. Franco, G. Martinelli, L. Reina, Nucl. Phys. B 415 (1994) 403.

18. G. Buchalla, A. J. Buras, M. E. Lautenbacher, Rev. Mod. Phys. 68 (1996) 1125.

19. M. Misiak et al., Phys. Rev. Lett. 114 (2015) 22, 221801.

20. M. Czakon et al., JHEP 1504 (2015) 168.

21. C. Bobeth et al., Phys. Rev. Lett. 112 (2014) 101801.

22. R. S. Chivukula, H. Georgi, Phys. Lett. B 188 (1987) 99.

23. L. Wolfenstein, Phys. Rev. Lett. 51, 1945 (1983).

24. J. Charles et al. [CKMfitter Collaboration], Eur. Phys. J. C41, 1 (2005), online update at http://www.slac.stanford.edu/xorg/ckmfitter/

25. M. Bona et al. [UTfit Collaboration], JHEP 0610 (2006) 081, online update at http://www.utfit.org/

26. G. 't Hooft, NATO Sci. Ser. B 59 (1980) 135.

27. G. Isidori, Y. Nir, G. Perez, Ann. Rev. Nucl. Part. Sci. 60 (2010) 355.

28. L. J. Hall, L. Randall, Phys. Rev. Lett. 65 (1990) 2939.

29. G. D'Ambrosio, G. F. Giudice, G. Isidori, A. Strumia, Nucl. Phys. B 645 (2002) 155. 
30. A. J. Buras et al., Phys. Lett. B 500 (2001) 161.

31. R. Aaij et al. [LHCb Collaboration], Phys. Rev. Lett. 111 (2013) 101805.

32. S. Chatrchyan et al. [CMS Collaboration], Phys. Rev. Lett. 111 (2013) 101804.

33. The CMS and LHCb Collaborations, CMS-PAS-BPH-13-007, LHCb-CONF-2013-012.

34. A. L. Kagan, G. Perez, T. Volansky and J. Zupan, Phys. Rev. D 80 (2009) 076002.

35. R. Barbieri, G. Isidori, J. Jones-Perez, P. Lodone and D. M. Straub, Eur. Phys. J. C 71 (2011) 1725.

36. J. Adam et al. [MEG Collaboration], Phys. Rev. Lett. 110 (2013) 201801.

37. V. Cirigliano, B. Grinstein, G. Isidori and M. B. Wise, Nucl. Phys. B 728 (2005) 121.

38. B. Grinstein, V. Cirigliano, G. Isidori and M. B. Wise, Nucl. Phys. B 763 (2007) 35.

39. S. Davidson and F. Palorini, Phys. Lett. B 642 (2006) 72.

40. M. B. Gavela, T. Hambye, D. Hernandez and P. Hernandez, JHEP 0909 (2009) 038.

41. R. Alonso, G. Isidori, L. Merlo, L. A. Munoz and E. Nardi, JHEP 1106 (2011) 037.

42. Y. Kuno, Prog. Part. Nucl. Phys. 82 (2015) 1.

43. D. B. Kaplan, Nucl. Phys. B 365 (1991) 259.

44. K. Agashe, G. Perez and A. Soni, Phys. Rev. D 71 (2005) 016002.

45. S. Davidson, G. Isidori and S. Uhlig, Phys. Lett. B 663, 73 (2008).

46. B. Keren-Zur, P. Lodone, M. Nardecchia, D. Pappadopulo, R. Rattazzi and L. Vecchi, Nucl. Phys. B 867 (2013) 429.

47. R. Barbieri, G. Isidori and D. Pappadopulo, JHEP 0902 (2009) 029.

48. M. Redi and A. Weiler, JHEP 1111 (2011) 108.

49. R. Barbieri, D. Buttazzo, F. Sala and D. M. Straub, JHEP 1210 (2012) 040.

50. R. Barbieri, D. Buttazzo, F. Sala, D. M. Straub and A. Tesi, JHEP 1305 (2013) 069.

51. B. Grinstein, M. Redi and G. Villadoro, JHEP 1011 (2010) 067.

52. G. Altarelli and F. Feruglio, Rev. Mod. Phys. 82 (2010) 2701.

53. F. Feruglio, arXiv:1503.04071.

54. R. Zwicky and T. Fischbacher, Phys. Rev. D 80 (2009) 076009.

55. F. P. An et al. [Daya Bay Collaboration], Phys. Rev. Lett. 108 (2012) 171803.

56. J. K. Ahn et al. [RENO Collaboration], Phys. Rev. Lett. 108 (2012) 191802.

57. L. Michel and L. A. Radicati, Proc. of the Fifth Coral Gables Conference on Symmetry principles at High Energy, ed. by B. Kursunoglu et al., W. H. Benjamin, Inc. New York (1965); Annals Phys. 66 (1971) 758.

58. N. Cabibbo and L. Maiani, in Evolution of particle physics, Academic Press (1970), 50, App. I.

59. C. D. Froggatt, H. B. Nielsen, Nucl. Phys. B147 (1979) 277.

60. L. E. Ibanez and G. G. Ross, Phys. Lett. B 332 (1994) 100.

61. A. Anselm and Z. Berezhiani, Nucl. Phys. B 484 (1997) 97.

62. R. Barbieri, L. J. Hall, G. L. Kane and G. G. Ross, hep-ph/9901228.

63. Z. Berezhiani and A. Rossi, Nucl. Phys. Proc. Suppl. 101 (2001) 410.

64. P. F. Harrison and W. G. Scott, Phys. Lett. B 628 (2005) 93.

65. T. Feldmann, M. Jung and T. Mannel, Phys. Rev. D 80 (2009) 033003.

66. R. Alonso, M. B. Gavela, L. Merlo and S. Rigolin, JHEP 1107 (2011) 012.

67. E. Nardi, Phys. Rev. D 84 (2011) 036008.

68. R. Alonso, M. B. Gavela, D. Hernandez and L. Merlo, Phys. Lett. B 715 (2012) 194.

69. J. R. Espinosa, C. S. Fong and E. Nardi, JHEP 1302 (2013) 137.

70. R. Alonso, M. B. Gavela, G. Isidori and L. Maiani, JHEP 1311 (2013) 187.

71. G. F. Giudice, PoS EPS -HEP2013 (2013) 163.

72. G. Blankenburg, G. Isidori and J. Jones-Perez, Eur. Phys. J. C 72 (2012) 2126. 